\title{
The optical afterglows and host galaxies of three short/hard gamma-ray bursts ${ }^{\star}$
}

\author{
P. D’Avanzo ${ }^{1}$, D. Malesani ${ }^{2}$, S. Covino ${ }^{1}$, S. Piranomonte ${ }^{3}$, A. Grazian ${ }^{3}$, D. Fugazza ${ }^{1}$, R. Margutti ${ }^{1,4}$, V. D’Elia ${ }^{3}$, \\ L. A. Antonelli ${ }^{3}$, S. Campana ${ }^{1}$, G. Chincarini ${ }^{1,4}$, M. Della Valle ${ }^{5,6,7}$, F. Fiore ${ }^{3}$, P. Goldoni ${ }^{8,9}$, J. Mao $^{1}$, R. Perna ${ }^{10}$, \\ R. Salvaterra ${ }^{1}$, L. Stella ${ }^{3}$, G. Stratta ${ }^{11}$, and G. Tagliaferri ${ }^{1}$
}

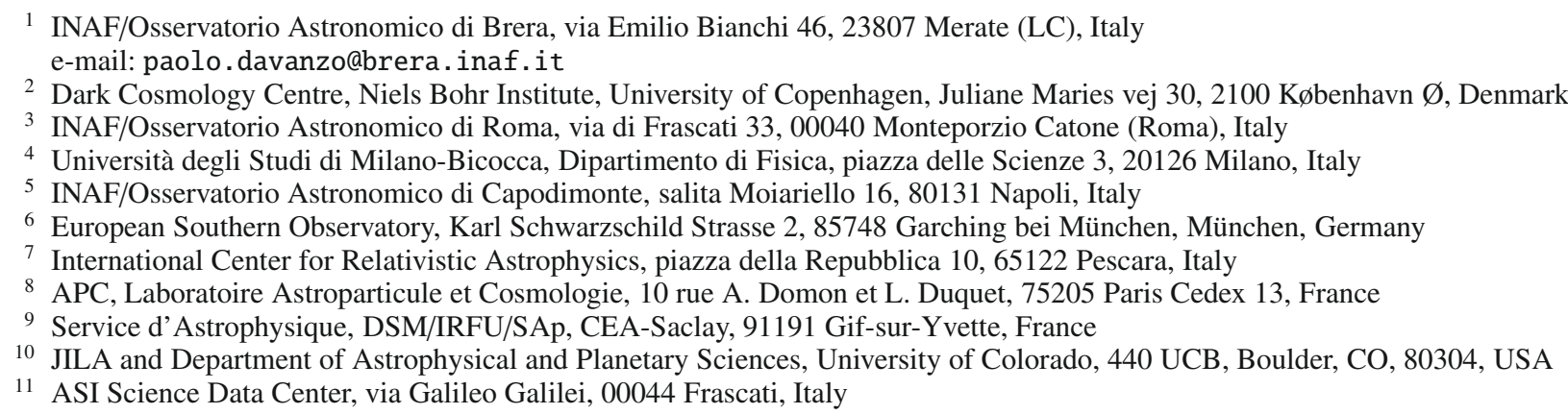

Received 5 November 2008 / Accepted 16 January 2009

\section{ABSTRACT}

\begin{abstract}
Context. Our knowledge of short gamma-ray bursts (GRBs) has significatively improved in the Swift era. Rapid multiband observations from the largest ground-based observatories led to the discovery of the optical afterglows and host galaxies of these events. In spite of these advancements, the number of short GRBs with secure detections in the optical is still fairly small. Short GRBs are commonly thought to originate from the merging of double compact object binaries but direct evidence for this scenario is still missing. Aims. Optical observations of short GRBs allow us to measure redshifts, firmly identify host galaxies, characterize their properties, and accurately localize GRBs within them. Multiwavelength observations of GRB afterglows provide useful information on the emission mechanisms at work. These are all key issues that allow one to discriminate among different models of these elusive events. Methods. We carried out photometric observations of the short/hard GRB 051227, GRB 061006, and GRB 071227 with the ESO-VLT starting from several hours after the explosion down to the host galaxy level several days later. For GRB 061006 and GRB 071227 we also obtained spectroscopic observations of the host galaxy. We compared the results obtained from our optical observations with the available X-ray data of these bursts.

Results. For all the three above bursts, we discovered optical afterglows and firmly identified their host galaxies. About half a day after the burst, the optical afterglows of GRB 051227 and GRB 061006 present a decay significatly steeper than in the X-rays. In the case of GRB 051227, the optical decay is so steep that it likely indicates different emission mechanisms in the two wavelength ranges. The three hosts are blue star forming galaxies at moderate redshifts and with metallicities comparable to the Solar one. The projected offsets of the optical afterglows from their host galaxy center span a wide range, but all afterglows lie within the light of their hosts and present evidence for local absorption in their X-ray spectra. We discuss our findings in light of the current models of short GRB progenitors.
\end{abstract}

Key words. gamma rays: bursts

\section{Introduction}

Gamma-ray bursts (GRBs) are rapid, intense flashes of gammaray radiation. Their cosmological nature was first suggested by their isotropic distribution in the sky (Hakkila et al. 1994), and spectacularly confirmed by the detection of longwavelength (X-rays to radio ranges) afterglows (Costa et al. 1997; van Paradijs et al. 1997; Frail et al. 1997; Bremer et al. 1998; Heng et al. 2008). The final demonstration was provided by the measurement of cosmological redshifts (Metzger et al. 1997). Nowadays, these sources can be monitored up to weeks

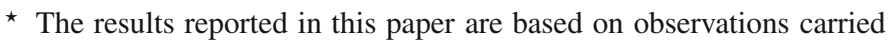
out at ESO telescopes under programmes Id 076.A-0392, 078.D-0809 and $080 . \mathrm{A}-0825$. and months after the explosion. Two classes of GRBs are known to date, with a classification based on their duration and spectral hardness (Kouveliotou et al. 1993). Short GRBs (with a duration $\$ 2$ s) are on average harder than long ones (typically lasting 10100 s). Although our knowledge of GRBs has significantly improved in recent years, optical and near-infrared (NIR) studies of the afterglows and host galaxies of short bursts are still limited to a small sample (e.g. Fox et al. 2005; Hjorth et al. 2005b; Berger et al. 2005; Castro-Tirado et al. 2005; Bloom et al. 2006; Covino et al. 2006; Levan et al. 2006; see Nakar 2007, for a review). While it has been firmly established that long GRBs (or at least a significant fraction of them) originate in core-collapse supernova (SN) explosions, the nature of short GRB progenitors is still under debate. This is due, at least in part, to the fact that short GRBs 
Table 1. VLT observation log for GRB 051227.

\begin{tabular}{ccccccc}
\hline \hline $\begin{array}{c}\text { Mean time } \\
\text { (UT) }\end{array}$ & $\begin{array}{c}\text { Exposure time } \\
(\mathrm{s})\end{array}$ & $\begin{array}{c}\text { Time since GRB } \\
\text { (days) }\end{array}$ & $\begin{array}{c}\text { Seeing } \\
\left({ }^{\prime}\right)\end{array}$ & Instrument & Magnitude & Filter \\
\hline 2005 Dec. 28.23379 & $10 \times 240$ & 0.47874 & 1.3 & VLT/FORS1 & $24.55 \pm 0.11$ & $R$ \\
2005 Dec. 29.21398 & $11 \times 300$ & 1.45803 & 1.1 & VLT/FORS1 & $25.59 \pm 0.18$ & $R$ \\
2005 Dec. 31.25894 & $18 \times 360$ & 3.50299 & 0.9 & VLT/FORS1 & $25.49 \pm 0.09$ & $R$ \\
2006 Jan. 02.26486 & $20 \times 360$ & 5.50891 & 1.0 & VLT/FORS1 & $25.73 \pm 0.11$ & $V$ \\
2006 Jan. 06.27768 & $12 \times 300$ & 9.52173 & 1.0 & VLT/FORS1 & $26.06 \pm 0.15$ & $B$ \\
2006 Jan. 06.29822 & $6 \times 300$ & 9.54227 & 1.0 & VLT/FORS2 & $25.10 \pm 0.40$ & $I$ \\
2006 Mar. 30.07032 & $30 \times 4 \times 30$ & 92.31437 & 1.0 & VLT/ISAAC & $>23.1$ & $J$ \\
\hline
\end{tabular}

Magnitudes are in the Vega system and are not corrected for Galactic absorption. Errors and upper limits are given at $1 \sigma$ and $3 \sigma$ confidence level respectively.

are detected less frequently (about one tenth of the GRBs detected by Swift belong to the short class; Berger 2007) and have fainter afterglows (Kann et al. 2008). Short GRBs with known distances have isotropic-equivalent energies of $\sim 10^{48}-10^{52} \mathrm{erg}$, on average two orders of magnitude lower than those of long GRBs. Moreover, the association of several short GRBs with galaxies with low star formation (Gehrels et al. 2005; Berger et al. 2005; Malesani et al. 2007) and the tight upper limits on underlying SNe (Hjorth et al. 2005a; Fox et al. 2005; Covino et al. 2006; Kann et al. 2008) rule out models involving massive stellar progenitors. While the engine powering the burst may be similar for the short and long category, namely a black hole surrounded by an accretion torus, these observations strongly indicate different stellar progenitors (for recent reviews, see Lee \& Ramirez-Ruiz 2007; Nakar 2007).

The merger of a double neutron star (DNS) or a black hole/neutron star (BH-NS) binary system is currently the leading model. In such systems, the delay between binary formation and merging is driven by the gravitational wave inspiral time (strongly dependent on the initial system separation). Some systems are thus expected to drift away from the starforming regions in which they formed, before merging takes place. Simulations (Belczynski et al. 2002, 2006) show that a large fraction of the merging events should take place outside, or in the outskirts, of galaxies. The density of the diffuse medium in these regions is expected to be low and give rise to fainter afterglows, setting in at later times than those of long GRBs (e.g. Vietri 2000; Panaitescu et al. 2001). A much faster evolutionary channel has been proposed (Belczynski \& Kalogera 2001; Perna \& Belczynski 2002; Belczynski et al. 2006), leading to merging in only $\sim 10^{6}-10^{7} \mathrm{yr}$, when most systems are still immersed in their star forming regions. The above scenarios are based on "primordial" binaries, i.e. systems that were born as binaries. Given the relatively small delay between formation and merging $(<1 \mathrm{Gyr})$, the redshift distribution of short GRBs that results from them should broadly follow that of the star formation, especially at low redshift. Alternatively, a sizeable fraction of DNSs may form dynamically by binary exchange interactions in globular clusters during their core collapse (Grindlay et al. 2006). The resulting time delay between star-formation and merging would be dominated by the cluster core-collapse time and thus be comparable to the Hubble time (Hopman et al. 2006). Both formation channels (primordial and dynamical) may be required to account for the redshift distribution of short GRBs detected by Swift (Salvaterra et al. 2008; Guetta \& Stella 2008). Also, some short GRBs may originate from different progenitors (such as hyperflares from soft gamma-ray repeaters; Hurley et al. 2005; Tanvir et al. 2005; Frederiks et al. 2007; Mazets et al. 2008) and display different afterglows, if any.
Key issues that could help in discriminating between the different theoretical scenarios summarized above are reliable redshift determinations, the study of the host galaxies properties, and accurate measurements of the spatial offsets between afterglows and host galaxy centers, over a sufficiently large sample of events. To date, an associated host galaxy candidate has been found for about half of the Swift short GRBs. In particular, almost all well localized short GRBs $(<5$ " error radius) have a candidate host galaxy inside their position error circle, but only for a dozen events with an observed optical afterglow could a firm GRB-galaxy association be established. Among those bursts with an optical (sub-arcsec) localization, only GRB 061201, GRB 070809, and GRB 080503 currently lack a secure host identification down to $R \sim 25-28$ (Stratta et al. 2007; Perley et al. 2008b,a).

In this paper we present the results of a detailed optical and NIR multiband study of three short bursts, GRB 051227 , GRB 061006 and GRB 071227 . Our discovery of their optical afterglows, together with deep optical imaging at late times, enabled us to firmly associate a host galaxy with each event. In Sects. 3-5 we present the analysis of our observations and of the available X-ray data for these bursts. In Sect. 6 we discuss the properties of the afterglows and host galaxies of these GRBs and compare them with current models for the progenitors of short bursts. Throughout the paper we assume a flat FriedmanRobertson-Walker cosmology with $H_{0}=71 \mathrm{~km} \mathrm{~s}^{-1} \mathrm{Mpc}^{-1}$, $\Omega_{\mathrm{m}}=0.27$ and $\Omega_{\Lambda}=0.73$. All errors are at the $90 \%$ confidence level, unless stated otherwise.

\section{Observations and data analysis}

We observed the fields of GRB 051227, GRB 061006 and GRB 071227 with the ESO Very large Telescope (VLT), using the FORS1, FORS2 and ISAAC instruments. All nights were clear, with seeing in the $0.6^{\prime \prime}-1.3^{\prime \prime}$ range. For GRB 051227 we observed the optical afterglow ( $R$-band imaging) and the host galaxy ( $B V R I J$-band imaging). For GRB 061006 we obtained $I$-band images of the optical afterglow, and photometric $(B V R I J K)$ and spectroscopic observations of the associated host galaxy. We carried out $R$-band imaging of the afterglow of GRB 071227 and optical spectroscopy of the associated host galaxy. In order to look for the possible rising of a supernova associated to GRB 071227, we monitored its host galaxy in the $R$-band until about 40 days after the burst. A complete log of our observations, together with the results of our analysis, is given in Tables 1-3. Image reduction was carried out following the standard procedures: subtraction of an averaged bias frame and division by a normalized flat frame. The photometric calibration was achieved by observing Landolt standard fields (on different 
Table 2. VLT observation log for GRB 061006.

\begin{tabular}{ccccccc}
\hline \hline $\begin{array}{c}\text { Mean time } \\
(\mathrm{UT})\end{array}$ & $\begin{array}{c}\text { Exposure time } \\
(\mathrm{s})\end{array}$ & $\begin{array}{c}\text { Time since GRB } \\
(\text { days })\end{array}$ & $\begin{array}{c}\text { Seeing } \\
\left({ }^{\prime \prime}\right)\end{array}$ & Instrument & Magnitude & Filter / grism \\
\hline 2006 Oct. 07.32063 & $10 \times 180$ & 0.62214 & 0.8 & VLT/FORS1 & $22.35 \pm 0.05$ & $I$ \\
2006 Oct. 08.30600 & $10 \times 180$ & 1.91352 & 0.8 & VLT/FORS1 & $22.94 \pm 0.07$ & $I$ \\
2006 Oct. 09.19919 & $7 \times 180$ & 2.50080 & 0.9 & VLT/FORS1 & $22.91 \pm 0.09$ & $I$ \\
2006 Oct. 08.77089 & $180+300+165$ & 2.07239 & 0.9 & VLT/FORS1 & $23.96 \pm 0.12$ & $R$ \\
2007 Feb. 17.65358 & $40 \times 3 \times 20$ & 133.95509 & 1.2 & VLT/ISAAC & $>21.2$ & $K$ \\
2007 Feb. 22.13000 & $20 \times 180$ & 138.43153 & 0.6 & VLT/FORS2 & $25.92 \pm 0.12$ & $B$ \\
2007 Mar. 10.07215 & $47 \times 3 \times 20$ & 154.37368 & 0.8 & VLT/ISAAC & $22.00 \pm 0.20$ & $J$ \\
2007 Mar. 15.02346 & $6 \times 180$ & 159.32499 & 0.9 & VLT/FORS1 & $24.56 \pm 0.07$ & $V$ \\
2007 Mar. 15.03891 & $3 \times 180$ & 159.34044 & 0.9 & VLT/FORS1 & $23.05 \pm 0.12$ & $I$ \\
\hline 2006 Oct. 09.31165 & $4 \times 2700$ & 2.613155 & 0.9 & VLT/FORS1 & - & 300 V+GG375 \\
\hline
\end{tabular}

Magnitudes are in the Vega system and are not corrected for Galactic absorption. Errors and upper limits are given at $1 \sigma$ and $3 \sigma$ confidence level respectively.

Table 3. VLT observation log for GRB 071227. Magnitudes are in the Vega system and are not corrected for Galactic absorption.

\begin{tabular}{ccccccc}
\hline \hline $\begin{array}{c}\text { Mean time } \\
(\mathrm{UT})\end{array}$ & $\begin{array}{c}\text { Exposure time } \\
(\mathrm{s})\end{array}$ & $\begin{array}{c}\text { Time since GRB } \\
(\text { days })\end{array}$ & $\begin{array}{c}\text { Seeing } \\
\left({ }^{\prime \prime}\right)\end{array}$ & Instrument & Magnitude & Filter/grism \\
\hline 2007 Dec. 28.13346 & $2 \times 120$ & 0.29055 & 0.7 & VLT/FORS2 & $23.24 \pm 0.08$ & $R$ \\
2007 Dec. 31.21576 & $3 \times 180$ & 3.37133 & 0.7 & VLT/FORS2 & $23.88 \pm 0.17$ & $R$ \\
2008 Jan 03.11081 & $5 \times 180$ & 6.26790 & 0.8 & VLT/FORS2 & $23.71 \pm 0.16$ & $R$ \\
2008 Jan. 07.09081 & $5 \times 180$ & 10.24790 & 0.8 & VLT/FORS2 & $24.02 \pm 0.21$ & $R$ \\
2008 Jan. 16.22578 & $5 \times 180$ & 19.38287 & 0.7 & VLT/FORS2 & $23.64 \pm 0.14$ & $R$ \\
2008 Jan. 18.09430 & $10 \times 180$ & 21.25139 & 0.8 & VLT/FORS2 & $23.91 \pm 0.19$ & $R$ \\
2008 Jan. 23.06066 & $10 \times 180$ & 26.21775 & 0.9 & VLT/FORS2 & $23.83 \pm 0.31$ & $R$ \\
2008 Feb. 06.10412 & $10 \times 180$ & 40.26121 & 1.1 & VLT/FORS2 & $23.69 \pm 0.13$ & $R$ \\
\hline 2007 Dec. 28.21424 & $4 \times 1800$ & 0.37133 & 0.7 & VLT/FORS2 & - & 300 +GG375 \\
\hline
\end{tabular}

Magnitudes from Dec. 31.2 onwards are computed within an annulus with a radius of about 1'.5 centered on the afterglow position, and are therefore not representative of the host galaxy. Errors are given at a $1 \sigma$ confidence level.

nights). Aperture and PSF-matched photometry were performed using SExtractor (Bertin \& Arnouts 1996) and DAOPhot within ESO-MIDAS $^{1}$, respectively. Astrometric solutions were computed against the USNO-B1.0 catalogue ${ }^{2}$. In addition to ordinary photometry, we also carried out image subtraction with the ISIS package (Alard \& Lupton 1998; Alard 2000), a useful tool to find and pinpoint variable sources in the field, even when blended with other objects.

Our spectra of the host galaxy of GRB 061006 and GRB 071227 were acquired with the $300 \mathrm{~V}$ grism $(11 \AA F W H M)$, covering the 4000-9000 $\AA$ wavelength range. We used in all cases a $1^{\prime \prime}$ slit, resulting in an effective resolution of $R=440$. The extraction of the spectrum was performed with the IRAF ${ }^{3}$ software package. Wavelength and flux calibration of the spectra were achieved using a helium-argon lamp and observing spectrophotometric stars. For both GRB 061006 and GRB 071227 , we accounted for slit losses by matching the flux-calibrated spectra to our photometry, which was possible through a simple rescaling (by a factor of 1.4 and 2.6, respectively). This shows that the derived spectral shape is robust.

The X-ray light curves and spectra of the three afterglows were obtained starting from Level 1 products (event lists)

\footnotetext{
${ }^{1}$ http://www.eso.org/projects/esomidas/

2 http://www.nofs .navy.mil/data/fchpix/

${ }^{3} I R A F$ is distributed by the National Optical Astronomy Observatories, which are operated by the Association of the Universities for Research in Astronomy, Inc., under cooperative agreement with the National Science Foundation.
}

processed by the Swift Data Center at NASA/GSFC ${ }^{4}$. They were further processed following the standard procedure using the latest HEAsoft release ${ }^{5}$ at the time of writing (2009 Jan.). The conversion from count rates to flux density was computed assuming an absorbed power-law spectral model. We refer to the upcoming paper by Margutti et al. (2009, in preparation) for details about the X-ray light curves and spectra extraction.

\section{GRB 051227}

GRB 051227 was discovered by the Swift coded mask Burst Alert Telescope (BAT) on 2005 Dec. 27, at $t_{0}=18: 07: 16$ UT. The BAT light curve shows a multi-peak structure, with a duration of $T_{90}=8.0 \pm 0.2 \mathrm{~s}$ and a fluence of $(2.3 \pm 0.3) \times 10^{-7} \mathrm{erg} \mathrm{cm}^{-2}$ in the $15-150 \mathrm{keV}$ band; the event was initially classified as a long burst (Barbier et al. 2005; Hullinger et al. 2005). Further analysis of the Swift/BAT data revealed that the spectral lag between the 25-50 and 100$350 \mathrm{keV}$ energy bands is negligible and that the overall shape of the BAT light curve of this burst is similar to that of the short/hard burst GRB 050724, with an initial hard spike followed several seconds later by a softer tail. These results indicate that GRB 051227 very likely belongs to the short-duration class (Barthelmy et al. 2005a). The initial peak of GRB 051227 was also detected by HETE-2. A joint analysis of the HETE2/FREGATE and Swift/BAT data revealed that GRB 051227 is a hard burst (Sakamoto et al. 2005). Swift/XRT began to observe

\footnotetext{
4 http://heasarc.gsfc.nasa.gov/docs/swift/sdc/

5 http://heasarc.gsfc.nasa.gov/docs/software/lheasoft/
} 


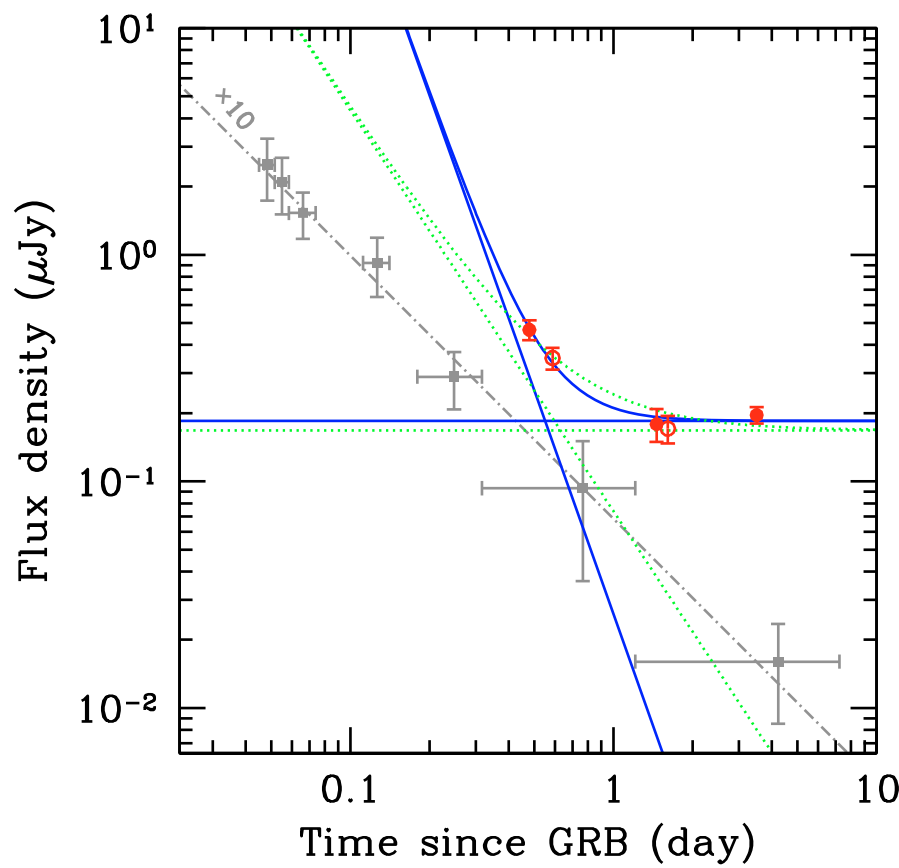

Fig. 1. $R$-band light curve of the GRB 051227 afterglow showing our VLT (filled circles) and Gemini (empty circles; from Berger et al. 2007a) data. The solid and dotted lines show the best power-law fit and the shallowest allowed decay (at 90\% confidence level), respectively. The squares show the X-ray flux density at $1 \mathrm{keV}$ (rescaled by a factor of 10), together with their best power-law fit (dot-dashed line). See Sect. 3.1 for details.

the GRB field $93 \mathrm{~s}$ after the BAT trigger and found a bright fading uncatalogued X-ray source. The X-ray light curve displayed a power-law like decay with small flares superposed (Beardmore et al. 2005). Optical ground-based observations, carried out at the ESO-VLT, revealed the presence of a variable source inside the XRT error box, identified as the optical afterglow of GRB 051227 (Malesani et al. 2005c,b,a; D’Avanzo et al. 2005). This was confirmed through Gemini observations (Soderberg \& Berger 2005). Further Gemini observations carried out on 2005 Dec. 30 revealed that the afterglow had faded below the host galaxy level (Berger \& Soderberg 2005). The afterglow was also detected in the $U$ band at the $4 \sigma$ level by Swift/UVOT with a magnitude $19.8 \pm 0.3$, but not in the other passbands (Roming et al. 2005). No radio counterpart was detected (Frail 2005).

\subsection{Results}

We started our observations of GRB $051227 \sim 0.5$ days after the burst, soon after it was recognized as a short/hard burst (Hullinger et al. 2005; Barthelmy et al. 2005a). In our first $R$-band image we found a point-like object inside the XRT error circle (Malesani et al. 2005a). In order to look for variability, we monitored the field at several epochs. Analysis of a second $R$-band observation, carried out on $2005 \mathrm{Dec}$. 29, revealed that our candidate had faded by $1.04 \pm 0.21$ mag with respect to our previous observation, confirming the source as the optical afterglow of GRB 051227 (Malesani et al. 2005b,c). Later $R$-band monitoring, carried out until Dec. 31 , showed no further variations of the brightness of the source, indicating that the host galaxy contribution was dominant (see Table 1 and Fig. 1). Given that we have only one detection of the afterglow in our light curve, we can only place a lower limit onto the slope of the flux decay. Assuming a power-law decay $F(t)=F_{0}+k t^{-\alpha}$, the decay index is constrained to be $\alpha>1.48$, and the host galaxy magnitude is $R=25.52_{-0.12}^{+0.18}$. In order to better constrain the decay slope, we added to our VLT data the Gemini-North observations reported by Berger et al. (2007a), converting their magnitudes from the $r$ band by adopting the conversion formulae recommended by the SDSS ${ }^{6}$. The combined best fit has $\alpha=3.27_{-1.50}^{+2.46}$ and the host magnitude is $R=25.55_{-0.12}^{+0.16}\left(\chi^{2} /\right.$ d.o.f. $=1.56 / 2$; Fig. 1). An analysis of all the available Swift/XRT data reveals an X-ray decay index significantly slower than the optical one around the same epoch. A power-law fit to the late points $(t>$ $2000 \mathrm{~s}$ ) of the $0.3-10 \mathrm{keV}$ light curve provides $\alpha_{\mathrm{X}}=1.16_{-0.25}^{+0.17}$ $\left(\chi^{2} /\right.$ d.o.f. $=1.16 / 5 ;$ Fig. 1$)$. An absorbed power-law fit to the X-ray spectrum gives a column density of $\left(2.3_{-0.9}^{+1.1}\right) \times 10^{21} \mathrm{~cm}^{-2}$ $\left(\chi^{2} /\right.$ d.o.f. $\left.=18.92 / 22\right)$, about a factor of 4 higher than the Galactic value in the direction of the burst. Image subtraction performed with the ISIS package confirmed the decay of the afterglow between our first two epochs of VLT observations and the flattening of the optical light curve (Fig. 2; D'Avanzo et al. 2005). Moreover, perfoming astrometry on the residual image of the subtraction process and on the images taken from Dec. 29 onwards, we could measure the accurate position of the afterglow inside the host galaxy. The afterglow has coordinates (J2000) $\mathrm{RA}=08^{\mathrm{h}} 20^{\mathrm{m}} 58^{\mathrm{s}} .10$, Dec $=+31^{\circ} 55^{\prime} 31^{\prime \prime} .9$ (0.'3 error). Its offset with respect to the galaxy center is $0.2 \pm 0.3$, negligible within the errors, and consistent with the constraint set by Berger et al. (2007a). Using their limit (which is tighter than ours), and remembering that the angular distance has a maximum at $z=1.65$ for our adopted cosmology, we infer a (projected) physical separation of less than $0.6 \mathrm{kpc}$ for any redshift. We note that the galaxy "G1" identified by Foley et al. (2005) is apparently not related to GRB 051227.

We carried out further multiband photometry of the host galaxy in the period 2005 Dec.-2007 Mar., obtaining $B$-, $V$-, $R$ - and $I$-band detections, and a deep $J$-band upper limit. The results of our photometry are reported in Table 2. The colors (corrected for Galactic extinction) are $B-V=0.29 \pm 0.19$, $V-R=0.16 \pm 0.23$ and $R-I=0.41 \pm 0.45$. Due to the faintness of the object, colors have large uncertainties, especially in the $I$ band, where detection is only marginal. Even with these large errors, the host galaxy of GRB 051227 appears very blue. Following Berger et al. (2007a), we can use the apparent luminosity of the host galaxy as a rough indicator of its redshift. The host galaxy, with an extinction-corrected magnitude $R \sim 25.4$, is quite faint and this could suggest a moderately high redshift. For comparison, we took a sample of galaxies with spectroscopic redshifts from the Gemini Deep Deep Survey (Abraham et al. 2004). We find that $90 \%$ of the systems in the magnitude range $24<R \lessgtr 25.5$ have redshift in the interval $0.8<z<2.4$. The host galaxy of GRB 051227 is actually at the faint end of the considered brightness range, hence it is probably at even higher redshift. Conservatively, we infer $z \gtrsim 0.8$ for this system. If real, the UVOT detection of the afterglow in the $U$ filter $^{7}$ $(\lambda=3500 \pm 500 \AA$ ) implies $z \lesssim 2.8$, otherwise the observed flux would be suppressed by the intergalactic hydrogen. In addition we note that, within the uncertainties of our photometry, the colors of the host galaxy are consistent with those of an irregular galaxy at $z \sim 0.8$ (Fukugita et al. 1995), in line with our estimates. At this redshift, the rest-frame $B$-band absolute magnitude of the host would be $M_{B}=-17.2 \mathrm{mag}$ (or $L_{B} \sim 0.03 L^{*}$,

\footnotetext{
6 http://www . sdss.org/dr6/algorithms/

sdssUBVRITransform. html\#Lupton2005

7 http://www.swift.ac.uk/filters. shtml
} 


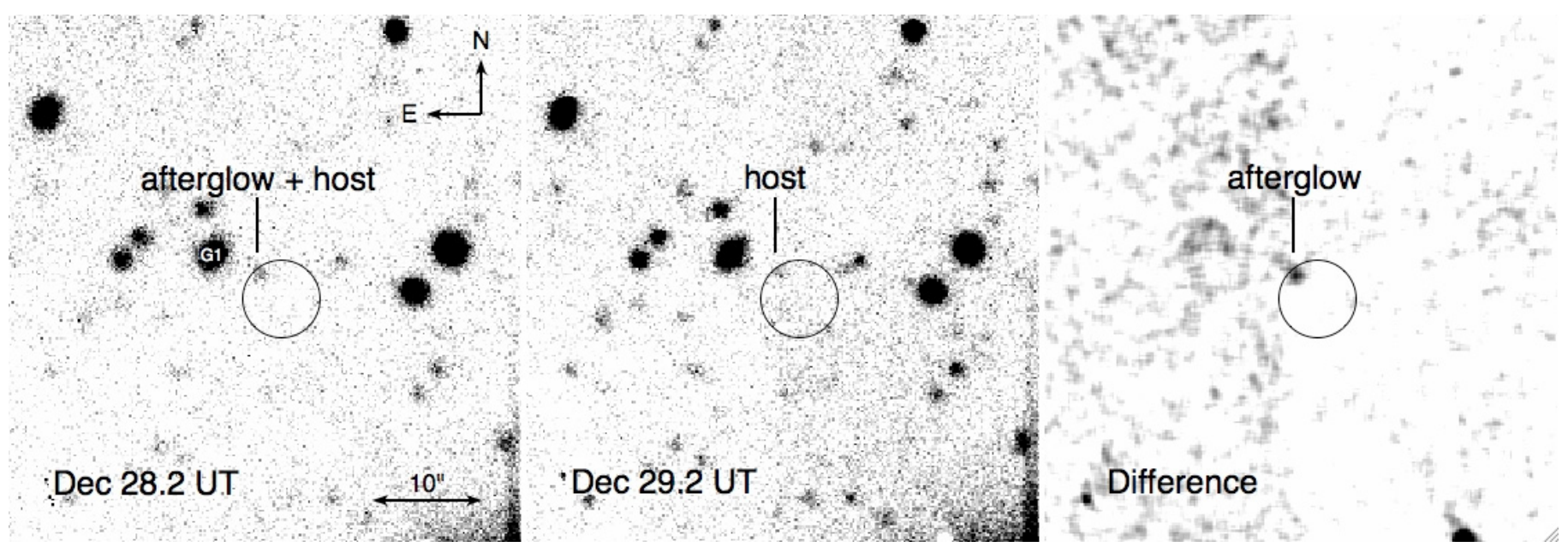

Fig. 2. $R$-band images of the field of GRB 051227 observed with the VLT about 0.5 (left) and 1.5 (middle) days after the burst. The fading of the afterglow is clearly visible in the subtraction image (right), which was smoothed for presentation purposes. The circle shows the X-ray position error (Beardmore et al. 2005). The galaxy G1, identified by Foley et al. (2005) and probably unrelated to the GRB, is marked in the left panel. See Sect. 3.1 for details.

assuming the absolute $B$-band magnitude of field galaxies to be $\left.M_{B}^{*}=-21\right)$, and the GRB isotropic-equivalent energy would be $E_{\gamma, \text { iso }}=3.9 \times 10^{50} \mathrm{erg}(15-150 \mathrm{keV})$.

\section{GRB 061006}

Swift triggered GRB061006 on 2006 Oct 06 at $t_{0}=$ 16:45:50 UT. The initial BAT light curve shows an impulse lasting about $5 \mathrm{~s}$. The burst was initially classified as long (Schady et al. 2006). At the same time, RHESSI, Konus/Wind and Suzaku detected a short burst at the same coordinates, with a duration $\leq 1 \mathrm{~s}$ (Hurley et al. 2006). A refined analysis of the BAT data revealed that the burst began with an intense double spike at $t \sim$ $t_{0}-22.5 \mathrm{~s}$ followed by a softer persistent emission lasting at least until $t \sim t_{0}+110 \mathrm{~s}$. The fluence in the $15-150$ and $20-2000 \mathrm{keV}$ bands was $(1.43 \pm 0.14) \times 10^{-6}$ and $3.6_{-1.9}^{+0.3} \times 10^{-6} \mathrm{erg} \mathrm{cm}^{-2}$, respectively (Krimm et al. 2006; Golenetskii et al. 2006). Swift/XRT began observing $143 \mathrm{~s}$ after the BAT trigger and found a faint fading X-ray source inside the BAT error circle (Schady et al. 2006). The 0.3-10 keV light curve showed a broken power-law decay (Troja et al. 2006a). No afterglow candidate was found with UVOT down to $18.5 \mathrm{mag}$ (white light) in the prompt images at $t_{0}+140 \mathrm{~s}$ (Schady et al. 2006). Optical ground-based observations, carried out at the ESO-VLT 0.6 and 1.9 days after the burst, revealed the presence of a variable source inside the XRT error box, identified as the optical afterglow of GRB 061006 (Malesani et al. 2006a,b).

\subsection{Results}

We started observing the field of GRB 061006 about 0.6 days after the burst, as soon as it became visible from the VLT. Given the rather high Galactic reddening $E(B-V)=0.32 \mathrm{mag}$, we initially decided to adopt the $I$ filter. In our first VLT image we found a point-like object inside the XRT error circle (Troja et al. 2006b). To search for variability, we continued monitoring the field at several epochs. Analysis of a second I-band observation, carried out on 2006 Oct. 8 (i.e. 1.9 days after the burst), revealed that the object inside the XRT error circle had faded by $0.59 \pm 0.09$ mag with respect to our previous observation, confirming that this source was the optical afterglow of GRB 061006

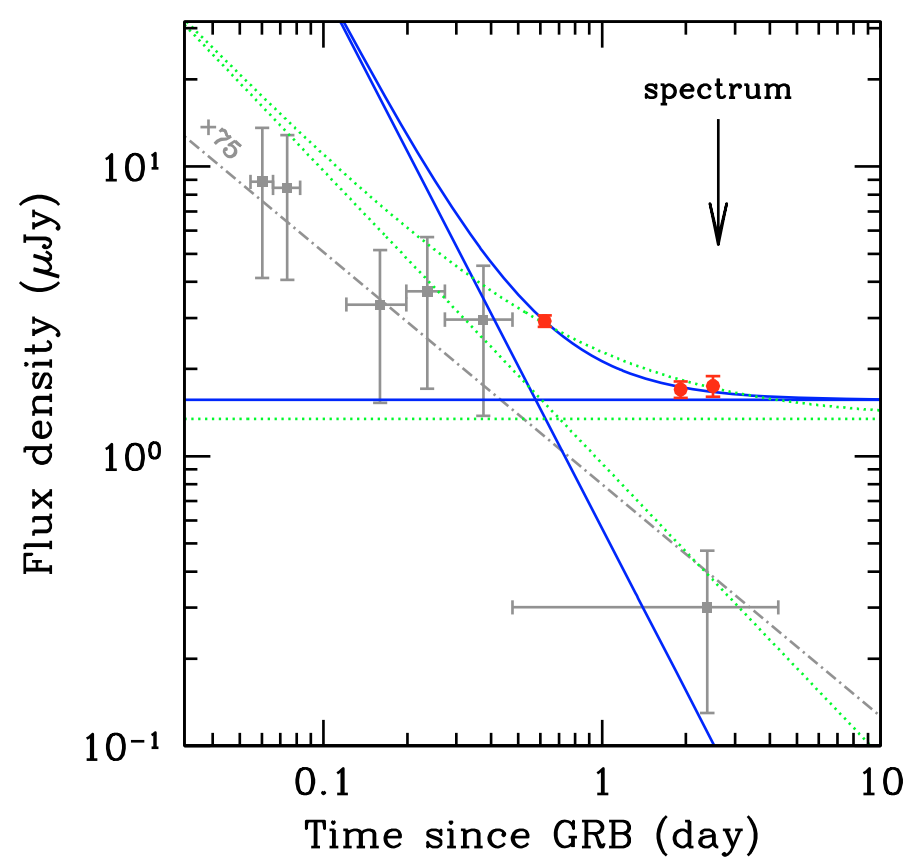

Fig. 3. $I$-band light curve of the GRB 061006 afterglow showing our VLT data (filled circles). The solid and dotted lines show the best power-law fit $\left(F(t)=F_{0}+k t^{-\alpha}\right)$ and the shallowest allowed decay, respectively. The squares show the X-ray flux density at $1 \mathrm{keV}$ (rescaled by a factor of 75), together with their best power-law fit (dot-dashed line). The arrow indicates the epoch of our spectrum. See Sect. 4.1 for details.

(Malesani et al. 2006a,b). Later I-band monitoring showed no further variations of the brightness of the source, indicating that the afterglow had faded below the host galaxy level (Table 2 and Fig. 3). Given that we have only one detection of the afterglow in our light curve, we can only set a lower limit to the decay slope. Assuming a power-law like decay $\left(F(t)=F_{0}+k t^{-\alpha}\right)$, the decay index is constrained to be $\alpha>1.01$ (with a best fit solution $\alpha=1.86 ; \chi^{2} /$ d.o.f. $\left.=0.43 / 1\right)$, while the host galaxy magnitude is $I=23.03_{-0.15}^{+0.22}$ (Fig. 3). Using the available X-rays data, we obtained the $0.3-10 \mathrm{keV}$ light curve and spectrum of the afterglow. We performed a power-law fit to the late points $(t>300 \mathrm{~s})$ 


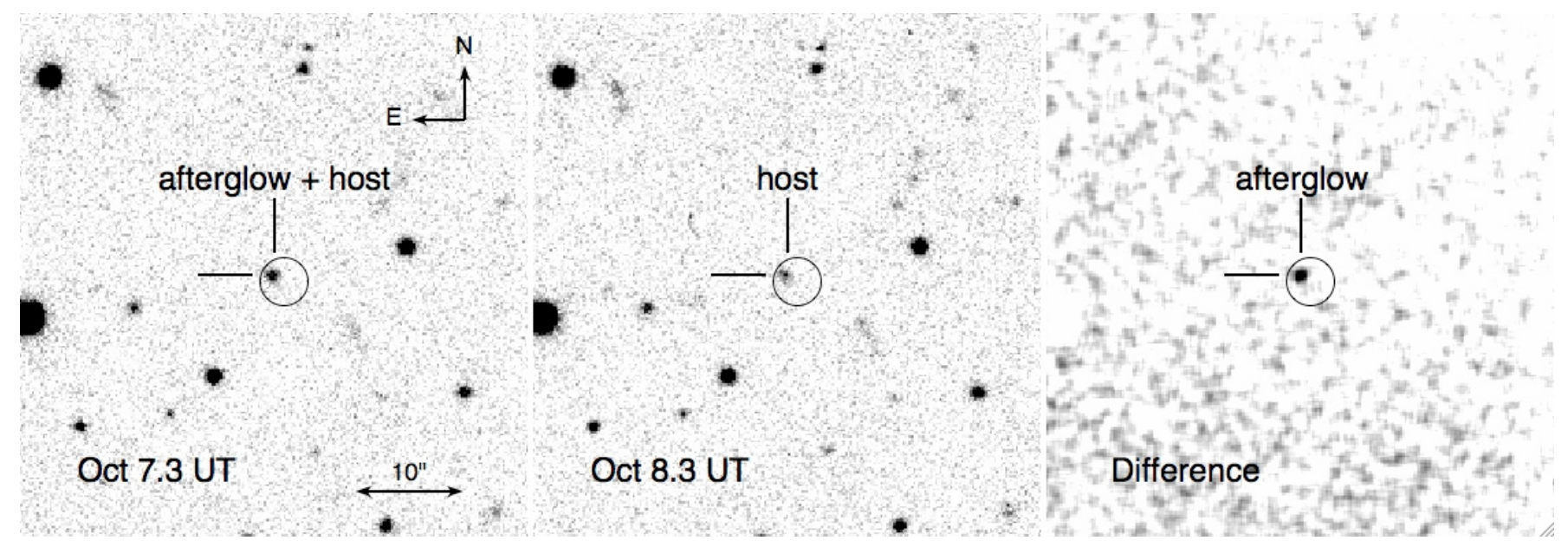

Fig. 4. I-band images of the field of GRB 061006 observed with the VLT about 0.6 (left) and 1.9 (middle) days after the burst. The fading of the afterglow is clearly visible in the subtraction image (right), which was smoothed for presentation purposes. The circle shows the X-ray position uncertainty (Troja et al. 2006b). See Sect. 4.1 for details.

obtaining a decay index of $\alpha_{\mathrm{X}}=0.80_{-0.15}^{+0.13}\left(\chi^{2} /\right.$ d.o.f. $\left.=1.74 / 7\right)$. We note that the optical decay is steeper than the X-ray decay at the same epoch. The X-ray spectrum of the afterglow can be fitted with an absorbed power law with an absorbing column comparable to the (relatively large) Galactic value in the direction of the burst.

Image subtraction performed with the ISIS package confirmed the decay of the afterglow between the first two epochs (Fig. 4) and the flattening of the optical light curve. As in the case of GRB 051227, we measured the positions of the GRB 061006 afterglow inside its host galaxy in the residual image of the subtraction process. The optical afterglow coordinates are (J2000): $\mathrm{RA}=07^{\mathrm{h}} 24^{\mathrm{m}} 07^{\mathrm{s}} \cdot 60$, Dec $=-79^{\circ} 11^{\prime} 55^{\prime \prime} .1$ ( 0.2 error $)$. The offset with respect to the host centre is $0 .{ }^{\prime} 3 \pm 0.0^{\prime} 3$.

On Oct. 9 we acquired a medium-resolution spectrum of the host galaxy. From the detection of several emission lines ([O II], $\mathrm{H} \beta$, [O III]), we derive a redshift $z=0.436 \pm 0.002$ (Fig. 5). This is consistent with the value reported by Berger (2007, 2009). The resulting rest-frame $B$-band absolute magnitude of the host is thus $M_{B}=-17.7$, or $L_{B} \sim 0.05 L^{*}$ (assuming $M_{B}^{*}=-21$ ). We secured further multiband photometry of the host galaxy in the period 2007 Feb.-2007 Mar., obtaining $B, V, R, I$ and $J$ detections, together with a deep $K$-band upper limit. All the results of our photometry are reported in Table 2 . The extinctioncorrected colors are $B-V=1.04 \pm 0.14, V-R=0.40 \pm 0.14$ and $R-I=0.78 \pm 0.14$, which are much bluer than those of elliptical galaxies and consistent with those of spirals (Sbc or Scd type) at $z \sim 0.5$ (Fukugita et al. 1995). This is in agreement with our spectroscopic redshift estimate. Considering the measured angular offset, at $z=0.436$ the afterglow is located within $3.5 \mathrm{kpc}$ (in projection) from the center of the host galaxy. At this redshift the isotropic-equivalent energy of GRB 061006 is $E_{\gamma \text {,iso }}=1.7 \times 10^{51} \mathrm{erg}(20-2000 \mathrm{keV})$.

As indicated from several emission lines, star formation is still ongoing in this galaxy. For the [O II] emission line, we measure a luminosity of $2.18 \times 10^{40} \mathrm{erg} \mathrm{s}^{-1}$ (corrected for slit losses). This converts to an unobscured star formation rate (SFR) of $0.3 M_{\odot} \mathrm{yr}^{-1}$ (Kennicutt 1998), which corresponds to a specific SFR of about $6.1 M_{\odot} \mathrm{yr}^{-1} L_{*}^{-1}$. This is comparable to the typical specific SFRs measured in long GRB host galaxies (Christensen et al. 2004).

The detection of oxygen and hydrogen emission lines in our spectrum allows us to determine the metallicity of the host

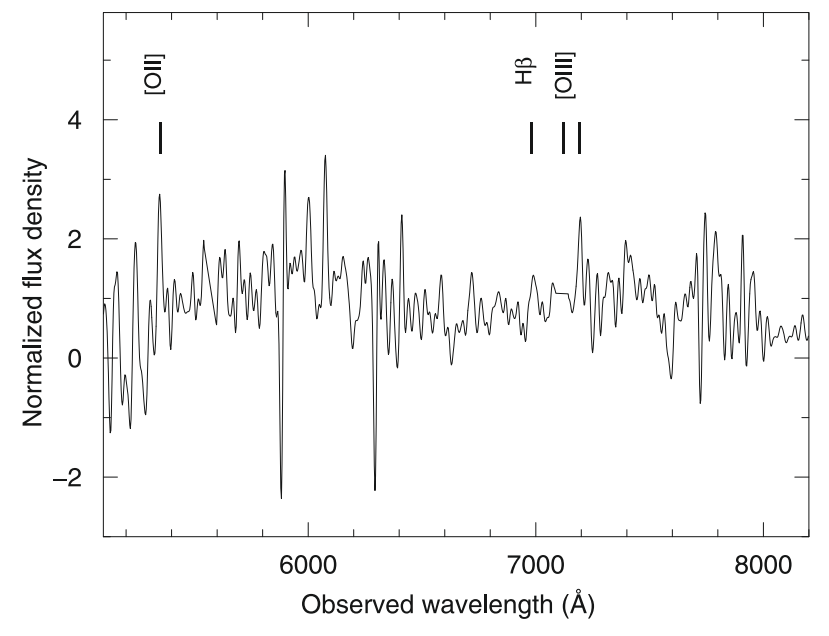

Fig. 5. VLT/FORS1 spectrum of the host galaxy of GRB 061006 at $z=0.436 \pm 0.002$.

galaxy. This can be done by adopting the commonly used abundance diagnostic $R_{23}=\left(F_{[\mathrm{OII}] \lambda 3727}+F_{[\mathrm{O} \text { III }] \lambda \lambda 4959,5007}\right) / F_{\mathrm{H} \beta}$. However, as can be seen in Fig. 5, the $\mathrm{H} \beta$ and [O III] $\lambda 4959$ emission lines have very low $S / N(\sim 2)$, which makes the $R_{23}$ parameter of little practical use. To overcome this problem, we used the empirical study performed by Nagao et al. (2006) on a large sample of galaxies, which shows that the ratio $F_{[\mathrm{OIII}] \lambda 5007} / F_{[\mathrm{OII}] \lambda 3727}$ can be used as a useful indicator of metallicity. For the host galaxy of GRB 061006 we obtain $F_{[\mathrm{OIII}] \lambda 5007} / F_{\text {[OII] } 3727}=0.52 \pm 0.23$ which corresponds, according to the study of these authors, to an oxygen abundance range $8.2 \leq 12+\log (\mathrm{O} / \mathrm{H}) \leq 8.9$, i.e. a metallicity in the range $(0.4-1.7) \times Z_{\odot}$ (using as solar abundance $12+\log (\mathrm{O} / \mathrm{H})=8.69$ from Allende Prieto et al. 2001). This is higher that what commonly found for the host galaxies of long GRBs (Savaglio et al. 2009).

\section{GRB 071227}

Swift triggered GRB 071227 on 2007 Dec. 27 at $t_{0}=$ 20:13:47 UT. A preliminary analysis of the BAT light curve revealed the presence of at least three pulses, with a total duration 


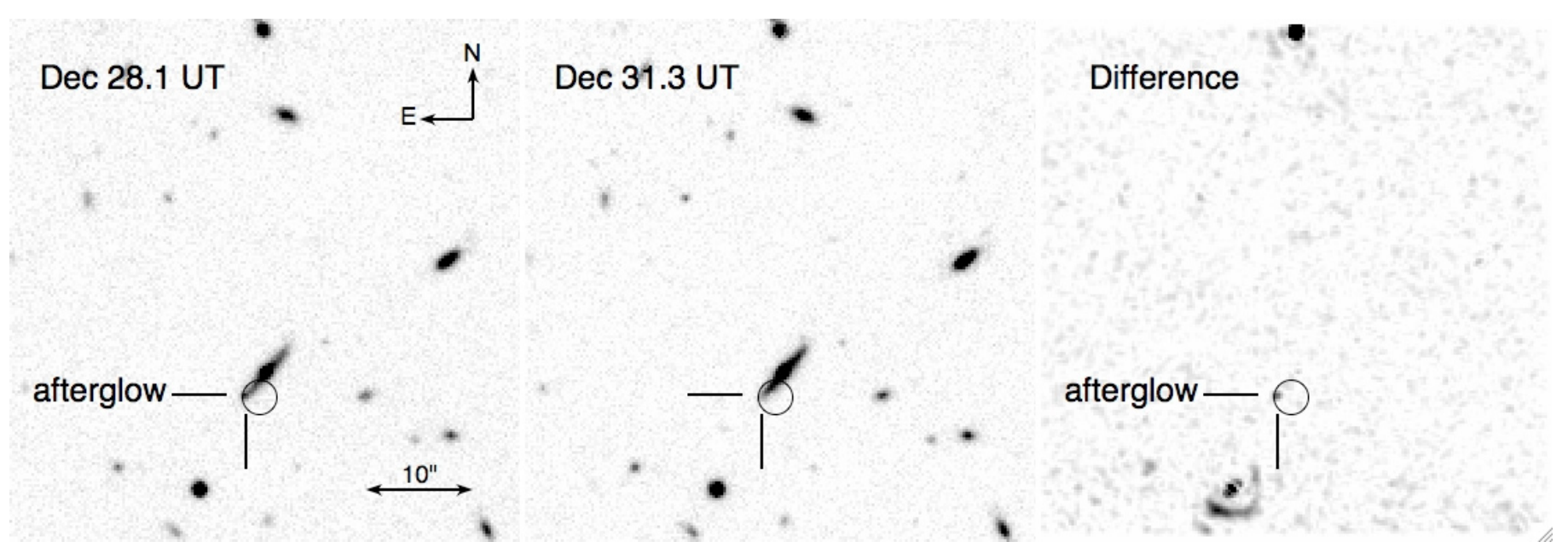

Fig. 6. $R$-band images of the field of GRB 071227 observed with the VLT about 0.3 (left) and 3.4 (middle) days after the burst. The fading of the afterglow is clearly visible in the subtraction image (right). The circle shows the X-ray position error (Beardmore et al. 2007). See Sect. 5.1 for details.

of about 5 s (Sakamoto et al. 2007). From a subsequent analysis of the BAT data in the $15-350 \mathrm{keV}$ range, the burst was classified as short/hard, with a multipeaked light curve with $T_{90}=1.8 \pm 0.4 \mathrm{~s}$ and an extended emission up to $t=t_{0}+$ $100 \mathrm{~s}$. The fluence in the $15-150$ and $20-1300 \mathrm{keV}$ bands was $(2.2 \pm 0.3) \times 10^{-7}$ and $(1.6 \pm 0.2) \times 10^{-6} \mathrm{erg} \mathrm{cm}^{-2}$, respectively (Sato et al. 2007; Golenetskii et al. 2007). Konus/Wind and Suzaku/WAM detected the prompt emission of GRB 071227; the analysis of their data confirmed the short/hard nature of this burst (Golenetskii et al. 2007; Onda et al. 2008). Swift/XRT began observing the GRB 071227 field $80 \mathrm{~s}$ after the BAT trigger and found a fading, uncatalogued X-ray source (Sakamoto et al. 2007). The $0.3-10 \mathrm{keV}$ light curve could be modelled with a double broken power-law (Beardmore et al. 2007). Swift/UVOT observations of the field of GRB 071227, carried out starting 86 $\mathrm{s}$ after the BAT trigger, revealed the presence of a source with a white-filter magnitude of 21.7 mag (Sakamoto et al. 2007; Cucchiara \& Sakamoto 2007), which was identified as an edgeon galaxy in ground-based observation with the Magellan telescope (Berger et al. 2007b). Further observations with the ESOVLT allowed measurement of the galaxy redshift $z=0.38$ and detection of a fading source at its edge, identified as the optical afterglow of GRB 071227 (D'Avanzo et al. 2007, 2008).

\subsection{Results}

We observed the field of GRB 071227 starting about 0.3 days after the burst. Our first $R$-band image clearly showed the presence of a disk galaxy, seen edge-on, overlapping with the XRT error circle, as already reported by Cucchiara et al. (2007) and Berger et al. (2007b). In addition, we also noted the presence of a bright spot in the SE edge of the galaxy, consistent with the XRT position. To check for variability, we took another image about 3 days later. The result of image subtraction between the two epochs is shown in Fig. 6 and reveals that our candidate clearly faded (D'Avanzo et al. 2008). The afterglow is superimposed on the plane of the host galaxy and accurate photometry is thus challenging. However, the image subtraction technique overcomes this problem. A "reference frame" (in this case, our first-epoch image) is subtracted from all the available images and photometry is performed on the residual images. For any variable object in the field the variation in flux, with respect to the reference frame, is given as output. Performing PSF photometry on the reference frame, it is possible to calibrate in magnitude the flux variations. The results of this procedure are reported in Table 3. Astrometry performed on the residual image of the subtraction procedure gives the following coordinates (J2000): $\mathrm{RA}=03^{\mathrm{h}} 52^{\mathrm{m}} 31^{\mathrm{s}} .23$, Dec $=-55^{\circ} 59^{\prime} 03^{\prime \prime} .2(0.3$ error $)$ for the optical afterglow, with a projected offset of $2 . \prime 9 \pm 0.9$ with respect to the host galaxy center (located at, $\mathrm{RA}=03^{\mathrm{h}} 52^{\mathrm{m}} 31^{\mathrm{s}} .02$, Dec $=-55^{\circ} 59^{\prime} 01^{\prime \prime} 0$ ). Further monitoring of the host galaxy with the ESO-VLT showed no more variation in brightness up to about 40 days after the burst. We measure a magnitude of $R \sim 20.6$ for the host galaxy. Assuming a power-law decay $\left(F(t)=F_{0}+k t^{-\alpha}\right)$, we can only constrain the decay index to be $\alpha>0.48$. This is due to the fact that we only have one datapoint during the afterglow phase. As expected for short bursts, in our light curve we find no evidence for the presence of a SN simultaneous to the GRB, with a $3 \sigma$ limit of $R=24.9$ (Fig. 7). Using the available X-ray data, we obtained the $0.3-10 \mathrm{keV}$ light curve and spectrum of the afterglow. A double broken power-law fit gives a decay index of $\alpha_{X}=0.95_{-0.16}^{+0.15}\left(\chi^{2} /\right.$ d.o.f. $\left.=83.40 / 70\right)$ around the epoch of our optical observations. The X-ray spectrum of the afterglow can be fitted with an absorbed power law with an absorbing column of $\left(1.6_{-0.6}^{+0.7}\right) \times 10^{21} \mathrm{~cm}^{-2}$, about 9 times higher than the Galactic value in that direction $\left(\chi^{2} /\right.$ d.o.f. $\left.=41.28 / 48\right)$.

On Dec. 27 we also carried out spectroscopic observations of the host galaxy of GRB 071227, with the medium-resolution grism 300V (Sect. 2). The slit was placed along the northsouth direction and centered on the nucleus of the galaxy. From the detection of several emission ([O II $],[\mathrm{O}$ III] and weak $\mathrm{H} \beta$ ) and absorption lines we derived a redshift $z=0.381 \pm 0.001$ (Fig. 8). This redshift was later confirmed by Berger et al. (2007). At the measured redshift, the isotropic-equivalent energy of GRB 071227 is $E_{\gamma \text {,iso }}=5.8 \times 10^{50} \mathrm{erg}(20-1300 \mathrm{keV})$, a value typical of short GRBs. According to the offset measured from our astrometry, at this redshift the afterglow is located at $15.0 \pm 2.2 \mathrm{kpc}$ from the center of the host galaxy. The host galaxy has a rest-frame $B$-band absolute magnitude $M_{B}=-19.3$, i.e. $L_{B} \sim 0.2 L^{*}$ (assuming $M_{B}^{*}=-21$ ). The presence of emission lines in the optical spectrum of the host galaxy of GRB 071227 indicates that star formation is ongoing in this system. We measure for the $[\mathrm{O} \mathrm{II}]$ emission line a luminosity of $3.9 \times 10^{40} \mathrm{erg} \mathrm{s}^{-1}$ (corrected for slit losses). This translates into an unobscured SFR 


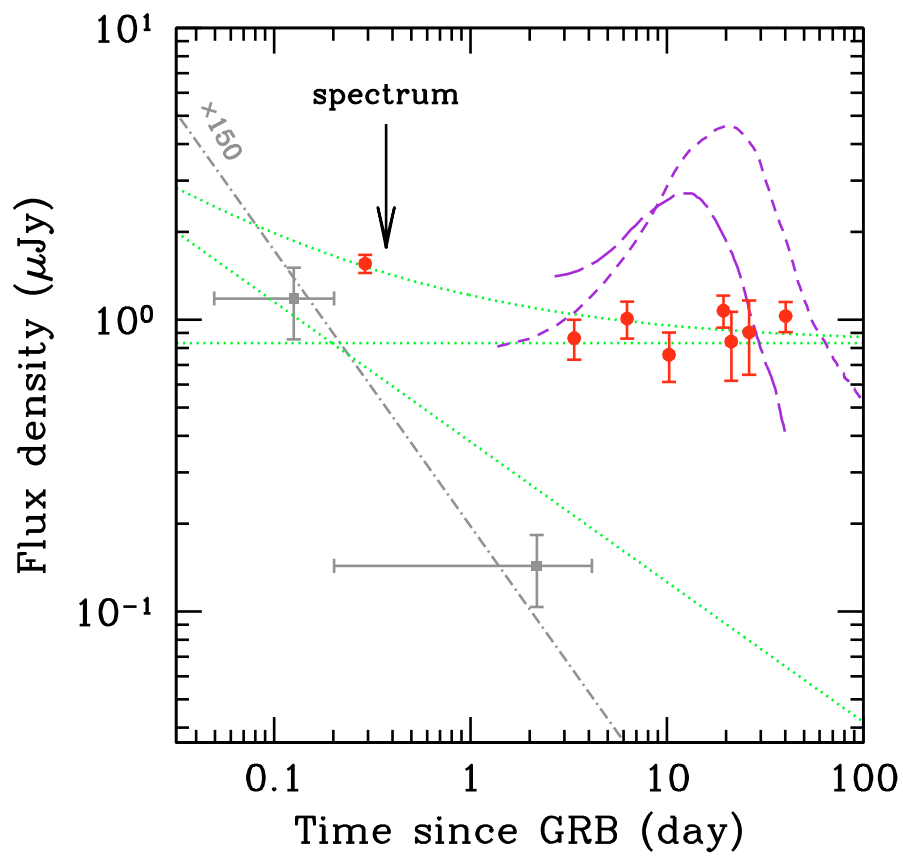

Fig. 7. $R$-band light curve of the GRB 071227 afterglow showing our VLT data (filled circles). The dotted line shows the shallowest allowed decay. The squares show the X-ray flux density at $1 \mathrm{keV}$ (rescaled by a factor of 150), together with their best power-law fit (dot-dashed line). The short- and long-dashed curves represent the light curves of SN 1998bw and SN2006aj (Galama et al. 1998; Pian et al. 2006) reported at the redshift of GRB $071227(z=0.381)$. The arrow indicates the epoch of our spectrum. Note that the optical data are computed inside an aperture of 1".5 radius, and therefore they do not represent the host galaxy brightness. See Sect. 5.1 for details.

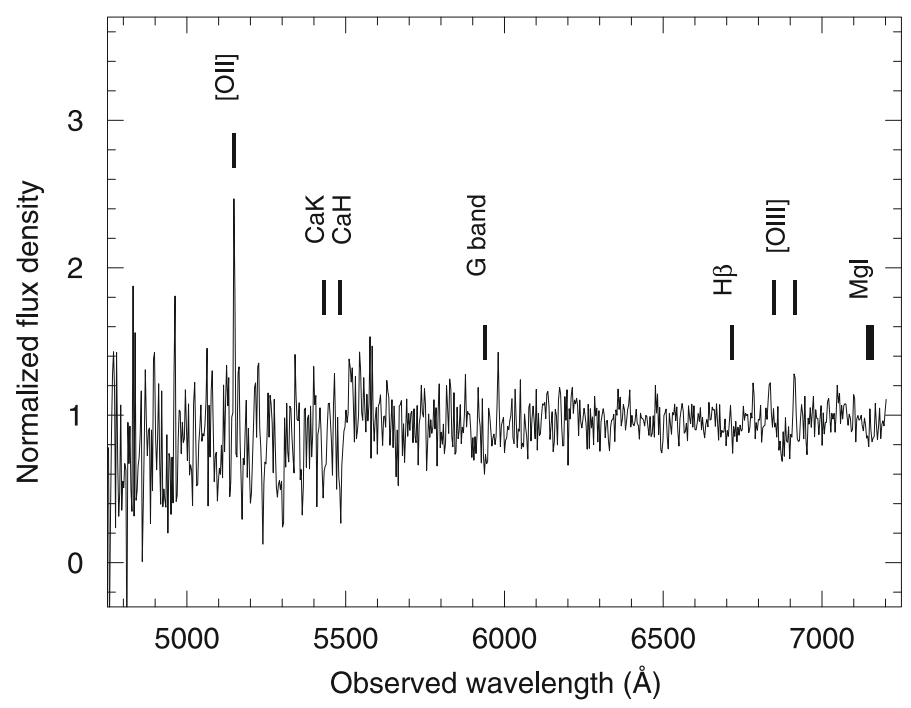

Fig. 8. VLT/FORS2 spectrum of the host galaxy of GRB 071227 at $z=$ $0.381 \pm 0.001$.

of $0.6 M_{\odot} \mathrm{yr}^{-1}$ (Kennicutt 1998), which corresponds to a specific SFR of about $2.7 M_{\odot} \mathrm{yr}^{-1} L_{*}^{-1}$. As we did for the host of GRB 061006 (Sect. 4.1), we can estimate the metallicity using the $F_{\text {[ОІг] }] \lambda 5007} / F_{\text {[ОІІ] } 3727}$ ratio as indicator (Nagao et al. 2006). We obtain a value of $0.58 \pm 0.13$ which corresponds to an oxygen abundance of $8.2 \leq 12+\log (\mathrm{O} / \mathrm{H}) \leq 8.8$, i.e. a metallicity in the range $(0.4-1.0) \times Z_{\odot}$, using the solar abundance from Allende Prieto et al. (2001). This estimate, similar to that obtained for the host galaxy of GRB 061006, is higher than those commonly found for the host galaxies of long GRBs.

\section{Discussion}

The set of short GRBs with detected optical afterglow is still very small, and the discovery of the optical counterparts for GRB 051227, GRB 061006 and GRB 071227 provides new cases with secure host galaxy identification. Analysis of the host properties provides substantial information on the progenitor systems. Furthermore, the study of afterglow evolution can also be used to investigate the emission processes of short GRBs.

\subsection{Afterglows}

Short GRB afterglow light curves have shown the same complexity observed in long-duration ones, including flares, breaks and plateau phases (e.g. Soderberg et al. 2006; de Ugarte Postigo et al. 2006; Roming et al. 2006; Stratta et al. 2007). It is not always straightforward to accomodate these features in the context of the standard model, but multiwavelength observations are required to meaningfully test it. While X-ray light curves are available for many of the Swift short GRBs, the optical evolution is rarely well enough sampled, if at all. A crucial issue is whether the emission in the two bands arises from the same process. The optical afterglows presented in this paper were too faint to allow an extensive monitoring, hence we cannot say much about the temporal evolution and the presence of breaks. However, both GRB 051227 and GRB 061006 present at $\sim 40-50 \mathrm{ks}$ a decay steeper in the optical than in the X-rays. This is similar to what has been observed in some long GRBs and it cannot be straightforward explained within the standard external shock model, where this behaviour occurs only in the case of a wind-shaped density profile. Such a medium is not expected around short GRB progenitors (Nakar 2007; Lee \& Ramirez-Ruiz 2007).

The decay of the GRB 051227 afterglow in the optical $(\alpha>$ 1.77 ) is actually particularly steep. Such indices are usually interpreted as evidence of jetted emission. In this case, however, the X-ray light curve would be expected to fall steeply as well, contrarily to what is observed. On the other hand, very steep optical decays have been observed for the afterglows of the short GRB 050724 and GRB 070707 (Berger et al. 2005; Malesani et al. 2007; Piranomonte et al. 2008). Also in these cases, the jet interpretation has been rejected, and the light curve shape has been explained assuming that long-lasting central engine activity was powering the afterglow emission. In particular, for GRB 050724 the optical emission was simultaneous to a large X-ray flare (Barthelmy et al. 2005b; Campana et al. 2006; Malesani et al. 2007), directly supporting late-time activity from the engine. Only sparse X-ray data are available for the INTEGRAL GRB 070707 (Piranomonte et al. 2008; McGlynn et al. 2008), but the observed optical decay index $\left(F(t) \propto\left(t-t_{0}\right)^{-\alpha}\right)$ is too steep if computed assuming as the time origin the GRB trigger instant. The only possible explanation is long-term activity from the central engine, so that the zero time $t_{0}$ is moved to a later epoch, and the decay index is shallower. We thus speculate that the rapid fading of GRB 051227 (and possibly of GRB 061006) could be due to an extra component visible in the optical, but not in X-rays. However, as mentioned in Sects. 3.1, 4.1 and 5.1, none of the GRBs presented in this work show flaring activity in their X-ray light curves at the time of our optical observations. 


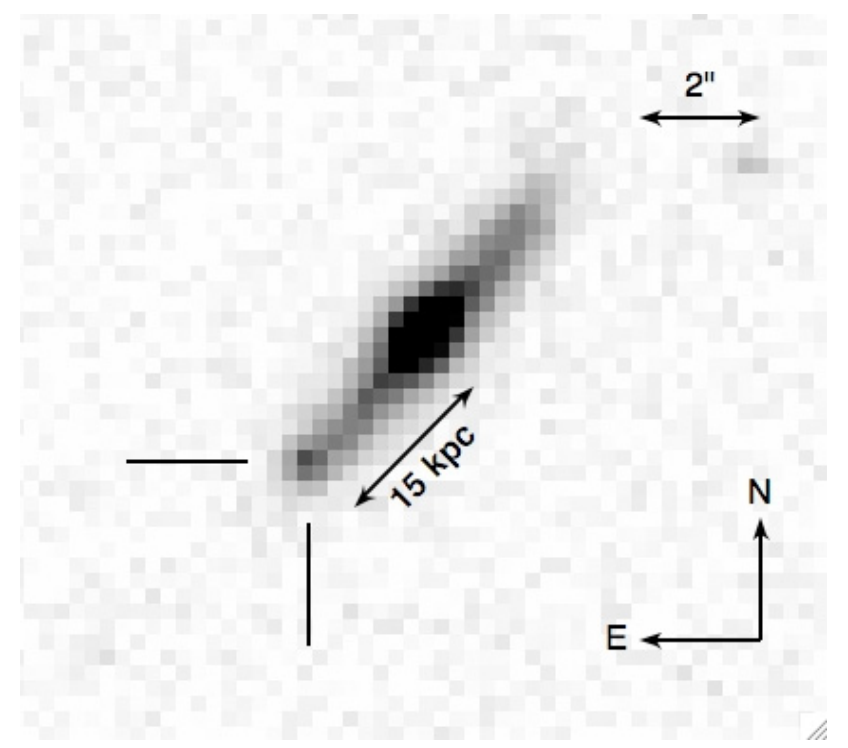

Fig. 9. Closing on the optical afterglow of GRB 071227 (marked by solid lines) and its host galaxy. The afterglow is located on top of the galaxy disk.

\subsection{Host galaxies: star formation rates and metallicities}

The blue colors of the GRB 051227 host suggest that active star formation is ongoing in this system. In the cases of GRB 061006 and GRB 071227, the star formation rate can be directly measured from the detection of [O II] in their optical spectra, at the level of about 6 and $3 M_{\odot} \mathrm{yr}^{-1} L_{*}^{-1}$, respectively. These values are comparable to the typical SFR observed in long GRB host galaxies (Christensen et al. 2004) and with those reported by Berger (2009) for a sample of nine short GRBs with a secure host galaxy association ${ }^{8}$. The presence of star forming activity in many short GRB host galaxies does not exclude a core-collapse origin for some of these events. With its relatively low redshift $(z=0.381)$ and well resolved host galaxy (Fig. 6), GRB 071227 is a perfect testing ground for this hypothesis. To this end, we carried out an intensive monitoring of the field of GRB 071227 (Fig. 7) up to about 40 days after the burst and we can exclude the presence of any associated supernova down to $M_{B}>-15.1$. With such limit we can exclude the association of GRB 071227 with a bright type-Ib/c SN like those observed in long-duration GRBs (Galama et al. 1998; Hjorth et al. 2003; Stanek et al. 2003; Malesani et al. 2004; Pian et al. 2006; Woosley \& Bloom 2006). This agrees with other results from the literature (Hjorth et al. 2005b; Covino et al. 2006; Kann et al. 2008). In a recent paper, Berger (2009) estimates metallicities for a sample of five short GRB host galaxies, in the range $(0.6-1.6) \times Z_{\odot}$. As reported in Sects. 4.1 and 5.1, we find similar values for the metallicities of the host galaxies of GRB 061006 and GRB 071227 . This shows that short GRBs explode inside relatively evolved stellar populations, putting an age constraint on the progenitor systems.

\subsection{Offsets}

As mentioned in Sect. 1, the study of the offset distribution of the afterglows with respect to their host galaxies is a key issue

\footnotetext{
8 With the significant exception of GRB 050724 and possibly GRB 050509B, the only short GRBs firmly associated with early-type galaxies and specific SFR lower than 0.01 and $0.03 M_{\odot} \mathrm{yr}^{-1} L_{*}^{-1}$ (Berger et al. 2005; Bloom et al. 2006).
}

in the study of short GRBs progenitors. In the context of double compact object progenitors, the offset distribution contains information on the merging times and thus on the evolutionary channels regulating binary systems evolution.

As reported in Sect. 5.1, the host galaxy of GRB 071227 is a disk galaxy, seen edge-on and has a radius of about $15 \mathrm{kpc}$, like the Milky Way. The GRB exploded on the galactic plane (Fig. 9), where probably most of the binary systems reside. Although we cannot exclude a chance superposition, its localization does not argue for a binary system progenitor born via binary exchange interactions in a globular cluster, given that most globular clusters are located in the galactic halos. The progenitor of GRB 071227 might thus be an example of the merging of a "primordial" binary system. The afterglow position of GRB 071227 lies right in the middle of the disc plane of its host galaxy, about $15 \mathrm{kpc}$ in projection away from the bulge. The large offset from the galaxy center could at first glance hint at a long delay time between the formation of the binary system and its merging ( 100 Myr; Belczynski et al. 2002, 2006). In this case, the binary would have enough time to travel the required distance. However, the afterglow is clearly lying on top of the galaxy light, hence any time delay can be accomodated. Indeed, in case of an ejected system, it would be fortuitous that the kick velocity vector was lying right in the galaxy plane. The binary system that generated GRB 071227 could indeed be a short-lived one, with an evolutionary channel that foresees a merging in only $\sim 10^{6}-10^{7}$ yr (Belczynski \& Kalogera 2001; Perna \& Belczynski 2002), or it may have received a negligible kick velocity. The system may thus still be embedded in its star forming region. Concerning this last possibility, we note that the X-ray spectrum of the afterglow of GRB 071227 reveals significant metal absorption (Sect. 5.1), indicating a dense medium. Also, evidence for local absorption rules against a merging occurred in a globular cluster, where the environment density is expected to be very low. These considerations highlight once more the role that spectroscopy of short GRB afterglows plays in revealing the nature of the progenitors of these events. Evidence for a dense medium is apparent also in the X-ray spectrum of the afterglow of GRB 051227 (Sect. 3.1). Even without an accurate redshift determination, we can set a limit of $0.6 \mathrm{kpc}$ on the projected offset between the GRB explosion site and the host galaxy center (Sect. 3.1). The host galaxy of GRB 061006 seems to be a starforming galaxy too (Sect. 4.1), but less luminous, and probably smaller, than the host galaxy of GRB 071227. In our VLT images we cannot spatially resolve the galaxy but, thanks to the image subtraction technique, we are able to constrain the afterglow offset with respect to its center, which is smaller than $3.5 \mathrm{kpc}$ (in projection). No excess column density has been found in the X-ray spectrum (Sect. 4.1), but the limits are not very constraining due to the large foreground component $\left(1.3 \times 10^{21} \mathrm{~cm}^{-2}\right)$. The small offsets and the presence of gas absorption point towards a "primordial" binary progenitor also for GRB 061006 and GRB 051227. In these cases too, an optical spectrum taken during the early stages of the afterglow could have given useful hints. Featureless spectra have recently been reported for the short bursts GRB 061201 (Stratta et al. 2007) and GRB 070707 (Piranomonte et al. 2008), but with a low signal-to-noise.

Finally, we note that the three short GRBs studied in this paper show an extended-duration soft emission component in their gamma-ray prompt light curves (Barthelmy et al. 2005a; Krimm et al. 2006; Sato et al. 2007) and, as said above, all of them are located within the light of their host galaxy. Recently, Troja et al. (2008) suggested that GRBs showing an extended-duration soft emission component preferentially have small offsets. This 
could be a consequence of a common physical progenitor $(\mathrm{BH}-$ NS binary), expected to merge on short timescales, which would lead to very little distance travelled before producing the GRB. Independently of its interpretation, such a conclusion has to be considered with some caution, given that not all the short GRBs have a secure host galaxy association (and, consequently, some of the offsets reported in the literature could be misleading; Piranomonte et al. 2008). Alternatively, as suggested by Bernardini et al. (2007), the presence of a temporally longlasting soft tail observed in short GRBs could be related to the density of the circumburst medium.

\section{Conclusions}

We have presented the results of a multiband optical and NIR observational campaign on the three short/hard GRBs GRB 051227, GRB 061006 and GRB 071227. We discovered their optical afterglows and identified their host galaxies.

The optical light curves of GRB 051227 and GRB 061006 show evidence for a steep decay between $\sim 0.5$ and $\sim 1.5$ days after the GRB, at variance with the shallow decay of their X-ray afterglows measured at similar epochs. These findings are difficult to explain based on the standard external shock model and, particularly for the case of GRB 051227, this behaviour can be interpreted as due to different mechanisms producing the optical and X-ray afterglows.

With our dataset we were able to characterize the host galaxies of these short GRBs. These galaxies are of late type, lie at relatively low redshifts and present a moderate level of star formation activity. The absence of brightening in the late optical light curve of GRB 071227 rules against an association with a bright core collapse SN, though the limit $M_{B}>-15$ on the peak absolute magnitude cannot exclude the presence of a core-collapse explosion characterized by low energy and very little content of ${ }^{56} \mathrm{Ni}$ in the ejecta (Della Valle et al. 2006; Tominaga et al. 2007). The offsets between the afterglows and the centers of their host galaxies may suggest that these short GRBs originated from the merging of double compact objects system likely of "primordial" origin. In particular, the location of GRB 071227 on the plane of a disk galaxy argues against ejection. The detection of an absorbing column in the X-ray spectrum may indeed indicate that the binary was not far from its star forming region. Looking at the near future, monitoring of the early stages of the afterglows of short GRBs, together with prompt optical spectroscopy, will likely shed light on many open issues on the origin of these events.

Acknowledgements. We thank the referee for his/her useful comments and suggestions. P.D.A. acknowledges the Italian Space Agency for financial support through the project ASI I/R/023/05. D.M. acknowledges the Instrument Center for Danish Astrophysics for financial support. The Dark Cosmology Centre is funded by the Danish National Research Foundation. We acknowledge the invaluable help from the ESO staff at Paranal in carrying out our target-ofopportunity observations. This work made use of data supplied by the Swift Data Center at NASA/GSFC.

\section{References}

Abraham, R. G., Glazebrook, K., McCarthy, P. J., et al. 2004, AJ, 127, 2455

Alard, C. 2000, A\&AS, 144, 363

Alard, C., \& Lupton, R. H. 1998, ApJ, 503, 325

Allende Prieto, C., Lambert, D. L., \& Asplund, M. 2001, ApJ, 556, L63

Barbier, L., Barthelmy, S., Beardmore, A., et al. 2005, GRB Coordinates Network, 4397, 1

Barthelmy, S., Gehrels, N., Norris, J., \& Sakamoto, T. 2005a, GRB Coordinates Network, 4401, 1

Barthelmy, S. D., Chincarini, G., Burrows, D. N., et al. 2005b, Nature, 438, 994
Beardmore, A., Burrows, D. N., Page, K., Marshall, F., \& Chester, M. 2005, GRB Coordinates Network, 4402, 1

Beardmore, A. P., Page, K. L., \& Sakamoto, T. 2007, GRB Coordinates Network, 7153, 1

Belczynski, K., \& Kalogera, V. 2001, ApJ, 550, L183

Belczynski, K., Bulik, T., \& Kalogera, V. 2002, ApJ, 571, L147

Belczynski, K., Perna, R., Bulik, T., et al. 2006, ApJ, 648, 1110

Berger, E. 2007, ApJ, 670, 1254

Berger, E. 2009, ApJ, 690, 231

Berger, E., \& Soderberg, A. M. 2005, GRB Coordinates Network, 4419, 1

Berger, E., Price, P. A., Cenko, S. B., et al. 2005, Nature, 438, 988

Berger, E., Fox, D. B., Price, P. A., et al. 2007a, ApJ, 664, 1000

Berger, E., Morrell, N., \& Roth, M. 2007b, GRB Coordinates Network, 7151, 1 Bernardini, M. G., Bianco, C. L., Caito, L., et al. 2007, A\&A, 474, L13

Bertin, E., \& Arnouts, S. 1996, A\&AS, 117, 393

Bloom, J. S., Prochaska, J. X., Pooley, D., et al. 2006, ApJ, 638, 354

Bremer, M., Krichbaum, T. P., Galama, T. J., et al. 1998, A\&A, 332, L13

Campana, S., Tagliaferri, G., Lazzati, D., et al. 2006, A\&A, 454, 113

Castro-Tirado, A. J., de Ugarte Postigo, A., Gorosabel, J., et al. 2005, A\&A, 439, L15

Christensen, L., Hjorth, J., \& Gorosabel, J. 2004, A\&A, 425, 913

Costa, E., Frontera, F., Heise, J., et al. 1997, Nature, 387, 783

Covino, S., Malesani, D., Israel, G. L., et al. 2006, A\&A, 447, L5

Cucchiara, A., \& Sakamoto, T. 2007, GRB Coordinates Network, 7150, 1

D'Avanzo, P., Covino, S., Malesani, D., et al. 2005, GRB Coordinates Network, 4413, 1

D’Avanzo, P., Fiore, F., Piranomonte, S., et al. 2007, GRB Coordinates Network, 7152,1

D’Avanzo, P., Covino, S., Fugazza, D., et al. 2008, GRB Coordinates Network, 7157,1

de Ugarte Postigo, A., Castro-Tirado, A. J., Guziy, S., et al. 2006, ApJ, 648, L83 Della Valle, M., Chincarini, G., Panagia, N., et al. 2006, Nature, 444, 1050

Foley, R. J., Bloom, J. S., Prochaska, J. X., et al. 2005, GRB Coordinates Network, 4409, 1

Fox, D. B., Frail, D. A., Price, P. A., et al. 2005, Nature, 437, 845

Frail, D. A. 2005, GRB Coordinates Network, 4415, 1

Frail, D. A., Kulkarni, S. R., Nicastro, L., Feroci, M., \& Taylor, G. B. 1997, Nature, 389, 261

Frederiks, D. D., Palshin, V. D., Aptekar, R. L., et al. 2007, Astron. Lett., 33, 19 Fukugita, M., Shimasaku, K., \& Ichikawa, T. 1995, PASP, 107, 945

Galama, T. J., Vreeswijk, P. M., van Paradijs, J., et al. 1998, Nature, 395, 670

Gehrels, N., Sarazin, C. L., O'Brien, P. T., et al. 2005, Nature, 437, 851

Golenetskii, S., Aptekar, R., Mazets, E., et al. 2006, GRB Coordinates Network, 5710,1

Golenetskii, S., Aptekar, R., Mazets, E., et al. 2007, GRB Coordinates Network, 7155,1

Grindlay, J., Portegies Zwart, S., \& McMillan, S. 2006, Nature Physics, 2, 116

Guetta, D., \& Stella, L. 2008, A\&A, in press, [arXiv:0811.0684]

Hakkila, J., Meegan, C. A., Pendleton, G. N., et al. 1994, ApJ, 422, 659

Heng, K., Lazzati, D., Perna, R., et al. 2008, ApJ, 681, 1116

Hjorth, J., Sollerman, J., Møller, P., et al. 2003, Nature, 423, 847

Hjorth, J., Sollerman, J., Gorosabel, J., et al. 2005a, ApJ, 630, L117

Hjorth, J., Watson, D., Fynbo, J. P. U., et al. 2005b, Nature, 437, 859

Hopman, C., Guetta, D., Waxman, E., \& Portegies Zwart, S. 2006, ApJ, 643, L91

Hullinger, D., Barbier, L., Barthelmy, S., et al. 2005, GRB Coordinates Network, 4400, 1

Hurley, K., Boggs, S. E., Smith, D. M., et al. 2005, Nature, 434, 1098

Hurley, K., Cline, T., Smith, D. M., et al. 2006, GRB Coordinates Network, 5702,1

Kann, D. A., Klose, S., Zhang, B., et al. 2008, [arXiv: 0804 . 1959]

Kennicutt, Jr., R. C. 1998, ARA\&A, 36, 189

Kouveliotou, C., Meegan, C. A., Fishman, G. J., et al. 1993, ApJ, 413, L101

Krimm, H., Barbier, L., Barthelmy, S., et al. 2006, GRB Coordinates Network, 5704,1

Lee, W. H., \& Ramirez-Ruiz, E. 2007, New J. Phys., 9, 17

Levan, A. J., Tanvir, N. R., Fruchter, A. S., et al. 2006, ApJ, 648, L9

Malesani, D., Tagliaferri, G., Chincarini, G., et al. 2004, ApJ, 609, L5

Malesani, D., Covino, S., Depagne, E., et al. 2005a, GRB Coordinates Network, 4412, 1

Malesani, D., Piranomonte, S., Antonelli, L. A., et al. 2005b, GRB Coordinates Network, 4407, 1

Malesani, D., Piranomonte, S., Antonelli, L. A., et al. 2005c, GRB Coordinates Network, 4404, 1

Malesani, D., Stella, L., Covino, S., et al. 2006a, GRB Coordinates Network, 5705,1

Malesani, D., Stella, L., D’Avanzo, P., et al. 2006b, GRB Coordinates Network, 5718, 1

Malesani, D., Covino, S., D’Avanzo, P., et al. 2007, A\&A, 473, 77 
Mazets, E. P., Aptekar, R. L., Cline, T. L., et al. 2008, ApJ, 680, 545 McGlynn, S., Foley, S., McBreen, S., et al. 2008, A\&A, 486, 405 Metzger, M. R., Djorgovski, S. G., Kulkarni, S. R., et al. 1997, Nature, 387, 878 Nagao, T., Maiolino, R., \& Marconi, A. 2006, A\&A, 459, 85

Nakar, E. 2007, Phys. Rep., 442, 166

Onda, K., Tashiro, M., Terada, Y., et al. 2008, GRB Coordinates Network, 7158,1

Panaitescu, A., Kumar, P., \& Narayan, R. 2001, ApJ, 561, L171

Perley, D. A., Bloom, J. S., Levan, A. J., et al. 2008a, GRB Coordinates Network, 7749,1

Perley, D. A., Bloom, J. S., Modjaz, M., et al. 2008b, GRB Coordinates Network, 7889,1

Perna, R., \& Belczynski, K. 2002, ApJ, 570, 252

Pian, E., Mazzali, P. A., Masetti, N., et al. 2006, Nature, 442, 1011

Piranomonte, S., D'Avanzo, P., Covino, S., et al. 2008, A\&A, 491, 183

Roming, P., Barbier, L., Trippico, M., \& Nousek, J. 2005, GRB Coordinates Network, 4411, 1

Roming, P. W. A., Vanden Berk, D., Pal'shin, V., et al. 2006, ApJ, 651, 985

Sakamoto, T., Atteia, J.-L., Ricker, G., et al. 2005, GRB Coordinates Network, 4403,1

Sakamoto, T., Baumgartner, W. H., Beardmore, A. P., et al. 2007, GRB Coordinates Network, 7147,
Salvaterra, R., Cerutti, A., Chincarini, G., et al. 2008, MNRAS, 388, L6

Sato, G., Barbier, L., Barthelmy, S. D., et al. 2007, GRB Coordinates Network, 7148,1

Savaglio, S., Glazebrook, K., \& LeBorgne, D. 2009, ApJ, 691, 182

Schady, P., Cummings, J. R., Guidorzi, C., et al. 2006, GRB Coordinates Network, 5707, 1

Soderberg, A. M., \& Berger, E. 2005, GRB Coordinates Network, 4414, 1

Soderberg, A. M., Berger, E., Kasliwal, M., et al. 2006, ApJ, 650, 261

Stanek, K. Z., Matheson, T., Garnavich, P. M., et al. 2003, ApJ, 591, L17

Stratta, G., D’Avanzo, P., Piranomonte, S., et al. 2007, A\&A, 474, 827

Tanvir, N. R., Chapman, R., Levan, A. J., \& Priddey, R. S. 2005, Nature, 438, 991

Tominaga, N., Maeda, K., Umeda, H., et al. 2007, ApJ, 657, L77

Troja, E., Page, K. L., Burrows, D. N., \& Schady, P. 2006a, GRB Coordinates Network, 5703, 1

Troja, E., Page, K. L., Gehrels, N., \& Burrows, D. N. 2006b, GRB Coordinates Network, 5723, 1

Troja, E., King, A. R., O’Brien, P. T., Lyons, N., \& Cusumano, G. 2008, MNRAS, 385, L10

van Paradijs, J., Groot, P. J., Galama, T., et al. 1997, Nature, 386, 686

Vietri, M. 2000, Astroparticle Physics, 14, 211

Woosley, S. E., \& Bloom, J. S. 2006, ARA\&A, 44, 507 